\title{
First-principle calculations of the exchange interaction characteristics for multilayer magnetic structures
}

\author{
Marina Mamonova ${ }^{1, \star}$ and Vladimir Prudnikov ${ }^{1, \star \star}$ \\ ${ }^{1}$ Omsk State University, pr. Mira, 55a, Omsk, 644047 Russia
}

\begin{abstract}
Magnetic multilayer structures which consist of the ferromagnetic layers separated by nonmagnetic layers with giant magnetoresistance (GMR) are widely used as spintronic devices and read heads of hard disks. In this work magnetic and exchange interaction characteristics of $\mathrm{Co} / \mathrm{Cu} / \mathrm{Co}, \mathrm{Ni} / \mathrm{Cu} / \mathrm{Ni}$ and $\mathrm{Fe} / \mathrm{Cr} / \mathrm{Fe}$ multilayer structures are studied by using VASP software package within the Projector Augmented Wave (PAW) method with generalized-gradient approximation (GGA PBE).The finite temperature magnetism of these structures was studied by Monte-Carlo simulations with the use of the exchange interaction parameters obtained from the ab initio results.
\end{abstract}

\section{Introduction}

The ultrathin films on the basis of the magnetic transition metals are used as a components for magnetic multilayer structures with giant magnetoresistance(GMR) which consist of the ferromagnetic layers separated by nonmagnetic layers [1]. These magnetic structures are widely used as read heads of hard disks, memory and spintronic devices [2]. At present, the development of modern computer technology and methods for solving large systems of equations makes it possible to obtain with high accuracy in the calculation of the properties of substances from the first principles of the characteristics of low-dimensional systems that are difficult or expensive to investigate in an experiment. The first-principles calculations of the electronic and crystal structure of magnetic materials are based on the spin theory of the density functional and the numerical solution of the system of Kohn-Sham equations. In this paper atomic structures, magnetic properties and exchange interaction characteristics of ferromagnetic films on $\mathrm{Cu}$ and $\mathrm{Cr}$ substrates are studied with the use of VASP (Vienna Ab-Initio Simulation Package) software package [3].

\section{Model and methods}

The layers of ferromagnetic atoms in systems $\mathrm{Co} / \mathrm{Cu} / \mathrm{Co}$, $\mathrm{Ni} / \mathrm{Cu} / \mathrm{Ni}$ and $\mathrm{Fe} / \mathrm{Cr} / \mathrm{Fe}$ are placed pseudomorphically on each side of the nonmagnetic slab to retain the inversion symmetry. The magnetic moment of atoms is directed collinearly along the $z$ axis. The substrate with 100 orientation surface plane is modeled by the three-layer slab. Supercells for BCC (Fe/Cr)and $\mathrm{FCC}(\mathrm{Ni} / \mathrm{Cu}$ and $\mathrm{Co} / \mathrm{Cu})$ systems are given in Fig. 1. We modeled the surface cell

\footnotetext{
^e-mail: MamonovaMV@omsu.ru
}

${ }^{\star}$ e-mail: prudnikv@univer.omsk.su using an one atom in monolayer to calculate the energy of film formation and four atoms in a monolayer to calculate the exchange integrals.

We carried out calculations with the use of VASP by means of the Projector Augmented Wave (PAW) method with PBE [4] approximation to describe the exchangecorrelation interactions. We calculated the optimized lat-

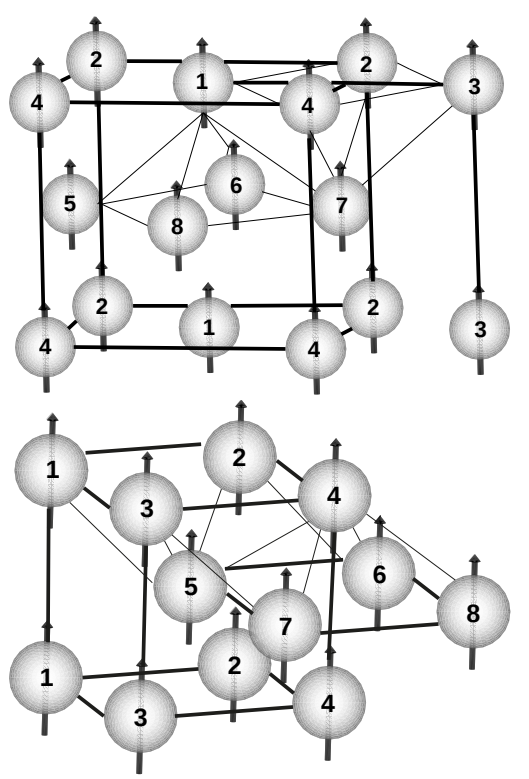

Figure 1. Atoms configurations in FCC and BCC super cells for three monolayers film. The arrows represent magnetic spins directions. The lines show connections the nearest and next-nearest neighbors atoms.

tice constant for bulk $\mathrm{Cu}$ through local density approximation (LDA) $a=3.524(1) \AA$ And generalized gradient approximation (GGA) $a=3.6367(5)$ calculations, that cor- 
Table 1. Results of calculation of the total $E_{\mathrm{tot}}$ and film formation $E_{\text {film }}$ energies and magnetic moment $\mu_{\text {tot }}$.

\begin{tabular}{llll}
\hline Position & $E_{\text {tot }}, \mathrm{eV}$ & $E_{\text {film }}, \mathrm{eV}$ & $\mu_{\text {tot }}, \frac{\mu_{b}}{\mathrm{at}}$ \\
\hline $\mathrm{Ni} / \mathrm{Cu}(100) \mathrm{fcc}$ & -27.588 & -1.405 & 0.330 \\
$\mathrm{Ni} / \mathrm{Cu}(111) \mathrm{fcc}$ & -28.118 & -1.159 & 0.430 \\
$\mathrm{Ni} / \mathrm{Cu}(111)$ bridge & -28.042 & -1.120 & 0.454 \\
$\mathrm{Ni} / \mathrm{Cu}(111)$ ontop & -27.347 & -0.774 & 0.531
\end{tabular}

responds to the value $a=3.6369 \AA$ Aobtained by other authors [5]. Also, the application of GGA approach for magnetic calculations is preferable due to more accurate band calculations and further accurate calculations of magnetic moments.

To determine the Ni film formation energy, it is necessary to calculate the difference between total energy of the system and the energy of its components:

$$
E_{\text {film }}=\frac{1}{2 n}\left(E_{N i C u}-E_{C u}-2 n E_{N i}\right)
$$

where $E_{C u}$ is the total energy of 5-ML Cu slab; $E_{N i C u}$ is the total energy of $\mathrm{Ni} / \mathrm{Cu} / \mathrm{Ni}$ system; $E_{N i}$ is the total energy of 1-ML Ni slab; $n$ is Ni film thickness in units of monolayers (we used one atom into the monolayer).

We obtained the energy of the film formation for $\mathrm{Ni} / \mathrm{Cu}(100) / \mathrm{Ni}$ system and the magnetic moment atom in 1-ML Ni slab as the functions of convergence parameters We confined ourselves to values of the plane waves cut-off energy $E_{\max }=350 \mathrm{eV}$ and Monkhorst Pack grid size $11 \times 11 \times 1$. Magnetic moments $\mu=1.02(7) \mu_{b}$ and $\mu=0.33$ (4) $\mu_{b}$, which were calculated correspondingly for atom free standing monolayer $\mathrm{Ni}$ film and for atom in 1ML Ni on $\mathrm{Cu}(001)$, are in good agreement with results $\mu=0.95 \mu_{b}$ and $\mu=0.37 \mu_{b}$ obtained by other authors[6].

Results of calculation of the total and film formation energies, interlayer distance relaxation parametes and magnetic moments are given in table 1. Comparison of the calculated film formation energies for various $\mathrm{Ni}$ atom positions (ontop, bridge, fcc) on $\mathrm{Cu}(111)$ substrate shows that more energetically favorable position is the position of repeating structure of the substrate. This position of the magnetic atoms are used in further calculations.

We considered the possibility of realization of the $\mathrm{Cu} / \mathrm{Ni} / \mathrm{Cu}(001)$ "sandwich" structure with two additional $\mathrm{Cu}$ cap layers, which are placed pseudomorphically on each side of the $\mathrm{Ni} / \mathrm{Cu}(001)$ slab. The calculations show that the energy of monolayer film system $E_{\text {tot }}=-27.134$ $\mathrm{eV}$ is greater than in the case of formation of the sandwich structure $E_{\text {tot }}=-28.287 \mathrm{eV}$. Therefore, these results determines that the formation of a sandwich structure is energetically more favorable.

The magnetic moment is the most fundamental quantity in magnetism and yet very difficult to measure experimentally in the case of thin films. The calculated values of the magnetic moments atoms at film layers and 1-st substrate layer for $\mathrm{Co} / \mathrm{Cu} / \mathrm{Co}, \mathrm{Ni} / \mathrm{Cu} / \mathrm{Ni}$ and $\mathrm{Fe} / \mathrm{Cr} / \mathrm{Fe}$ multilayer structures are presented in table 2.

Comparison of the magnetic moments of the atoms in different layers allows to conclude that the atoms most distant from the substrate have greatest magnetic moment.
Table 2. The calculated values of the $\mu_{0}$ - magnetic moment atom at 1-st substrate layer; $\mu_{i}$ - magnetic moment atom at $\mathrm{i}$ film layer; $\mu_{\mathrm{tot}}$ - average magnetic moment.

\begin{tabular}{llllll}
\hline & $\mu_{0}$ & $\mu_{1}$ & $\mu_{2}$ & $\mu_{3}$ & $\mu_{\text {tot }}$ \\
& $\mu_{B} / a t$ & $\mu_{B} / a t$ & $\mu_{B} / a t$ & $\mu_{B} / a t$ & $\mu_{B} / a t$ \\
\hline $\mathrm{Ni} / \mathrm{Cu} / \mathrm{Ni}$ & 0.014 & 0.489 & 0.678 & 0.776 & 0.622 \\
{$[7]$} & & 0.51 & 0.71 & 0.81 & \\
{$[8]$} & & $0.3(1)$ & $0.6(1)$ & $0.8(1)$ & \\
$\mathrm{Co} / \mathrm{Cu} / \mathrm{Co}$ & 0.024 & 1.602 & 1.580 & 1.825 & 1.670 \\
$\mathrm{Fe} / \mathrm{Cr} / \mathrm{Fe}$ & -0.318 & 2.084 & 2.417 & 2.932 & 2.349 \\
\hline
\end{tabular}

This regularity was also established both in experimental [8] and theoretical [7] investigations. Also, we can see in table 2 that the surface atoms of the nonmagnetic substrate are magnetized, and the directions of the magnetic moments for iron and chromium atoms are opposite in the $\mathrm{Fe} / \mathrm{Cr} / \mathrm{Fe}$ system.

\section{Methodology and results of calculation exchange interaction parameters}

The energy of quantum Heisenberg magnet per unit cell can be written as

$$
H=-\frac{1}{2 n} \sum_{i, j} J_{i j} S_{i} S_{j},
$$

where the summations are over the all sites in the unit cell, $S_{i}$ are quantum spin operators, $n$ is the number of unit cells in the crystal. The exchange interaction parameters of nearest neighbors and the next-nearest neighbors atoms $\left(d_{1}=(a \sqrt{2}) / 2\right.$ and $d_{2}=a$ for FCC structure as it is shown in Fig. 1) can be obtained from the difference of the total energy for the ferromagnetic and antiferromagnetic configurations of spins in the film:

$$
N_{i j} J_{j}=\Delta E_{i}=E_{i}^{\mathrm{AFM}}-E^{\mathrm{FM}},
$$

where $N_{i j}$ is a number of antiparallel pairs in one unit cell in the $i$-th AFM state for nearest neighbors $(j=1)$ and the next-nearest neighbors $(j=2)$ atoms. The values of parameters in this equation, obtained by our calculations are given in table 3-4. The considered antiferromagnetic configurations of spins are given in table 3. The sign '+' indicates the direction of the spin projection - upwards, the sign '-' the direction down. The order of alternation of signs corresponds to the numbering of atoms, an example of which is shown in Fig 1.

The investigation calculated values of the total energies for the ferromagnetic EF and antiferromagnetic EAF spin configurations of multilayer systems (Table 4) shows that, regardless of the system, a fully ordered ferromagnetic state is energetically more favorable.

\section{Results of calculations of the magnetoresistance}

On the basis of these values of the exchange interaction integrals, we calculated the temperature dependence of the 

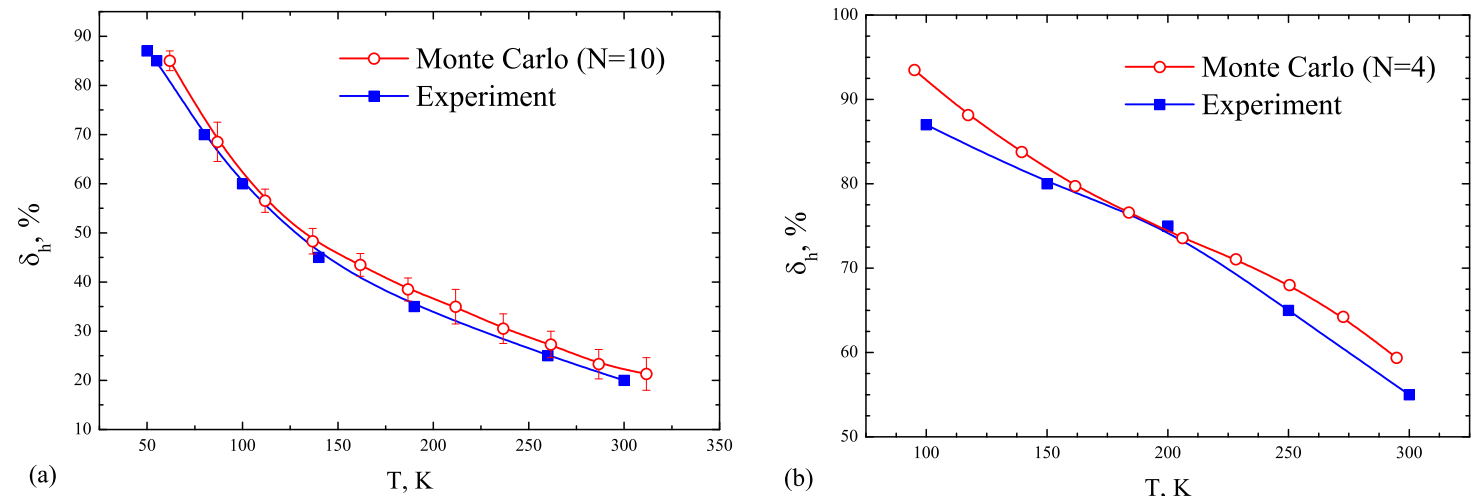

Figure 2. Comparison of the calculated and experimentally measured [1] temperature dependence of the magnetoresistance with the use of the ab initio determined values of the exchange integral for $\mathrm{Fe} / \mathrm{Cr} / \mathrm{Fe}(\mathrm{a})$ and $\mathrm{Co} / \mathrm{Cu} / \mathrm{Co}$ (b) structures.

Table 3. Antiferromagnetic spin configurations, the number of antiparallel pairs $N_{i j}$, and results of calculation of the i AFM states total energy $E A F_{i}$.

\begin{tabular}{|c|c|c|c|c|c|c|c|}
\hline & AFM state & & & & & $N_{1}$ & $N_{2}$ \\
\hline \multirow[t]{6}{*}{$\mathrm{BCC}$} & 1 & - & - & + & + & 20 & 24 \\
\hline & & + & + & - & - & & \\
\hline & & - & - & + & + & & \\
\hline & 2 & + & - & - & + & 24 & 12 \\
\hline & & - & + & + & - & & \\
\hline & & + & - & - & + & & \\
\hline \multirow[t]{6}{*}{$\mathrm{FCC}$} & 1 & + & - & + & - & 22 & 0 \\
\hline & & - & + & - & + & & \\
\hline & & + & - & + & - & & \\
\hline & 2 & + & + & - & - & 18 & 6 \\
\hline & & - & - & + & + & & \\
\hline & & + & + & - & - & & \\
\hline
\end{tabular}

Table 4. The calculated values of the total energies for the ferromagnetic EF and antiferromagnetic EAF spin configurations.

\begin{tabular}{llll}
\hline & $\begin{array}{l}\text { EF } \\
\text { eV }\end{array}$ & $\begin{array}{l}\text { EAF1 } \\
\text { eV }\end{array}$ & $\begin{array}{l}\text { EAF2 } \\
\text { eV }\end{array}$ \\
\hline $\mathrm{Ni} / \mathrm{Cu} / \mathrm{Ni}$ & -167.819 & -166.749 & -166.691 \\
$\mathrm{Co} / \mathrm{Cu} / \mathrm{Co}$ & -201.463 & -197.313 & -197.313 \\
$\mathrm{Fe} / \mathrm{Cr} / \mathrm{Fe}$ & -300.036 & -295.483 & -295.621 \\
\hline
\end{tabular}

Table 5. The calculated values of the exchange integrals of nearest neighbor and the next nearest neighbor interaction.

\begin{tabular}{lll}
\hline & $\begin{array}{l}J_{1} * 10^{-14}, \\
\text { erg }\end{array}$ & $\begin{array}{l}J_{2} * 10^{-14}, \\
\text { erg }\end{array}$ \\
\hline $\mathrm{Ni} / \mathrm{Cu} / \mathrm{Ni}$ & 0.65 & 0.56 \\
$\mathrm{Co} / \mathrm{Cu} / \mathrm{Co}$ & 2.52 & 1.68 \\
$\mathrm{Fe} / \mathrm{Cr} / \mathrm{Fe}$ & 1.73 & 0.73 \\
\hline
\end{tabular}

magnetoresistance using the Monte-Carlo simulations for the trilayer structures $\mathrm{Fe} / \mathrm{Cr}(100) / \mathrm{Fe}$ and $\mathrm{Co} / \mathrm{Cu}(100) / \mathrm{Co}$ with different thicknesses $N$ of ferromagnetic films. Magnetoresistance for a multilayer structures is introduced as $\delta_{h}=\left(R_{A P}-R_{P}\right) / R_{P}$, where $R_{A P}$ and $R_{P}$ are the resistance of the structure when the magnetizations of adjacent ferromagnetic layers are aligned antiparallel and parallel orientation of the magnetization of ferromagnetic layers respectively. The procedure of calculation consists of the following steps: the first step is connected with Monte Carlo simulations of a magnetic structure in the equilibrium state at temperature $\mathrm{T}$ with determination of the magnetization of ferromagnetic films $m_{1}$ and $m_{2}$, which gives the possibility of calculating the electron densities $n_{\uparrow \downarrow}$ for film cells; in the second step the average electron velocities $\mathrm{V}$ and the current densities $J_{i, \uparrow, \downarrow}=e V n_{\uparrow \downarrow}$ are calculated under relations

$$
\begin{gathered}
V=\mu E=\frac{e Z a^{2}}{k T} e^{-\frac{\Delta E_{i}}{k T}} E \\
E_{i, \uparrow, \downarrow}=\mp J_{1}\left[\sum_{j \neq i} S_{j}^{z}\left(n_{j, \uparrow}-n_{j, \downarrow}\right)+S_{i}^{z}\left(n_{i, \uparrow}-n_{i, \downarrow}\right)\right],
\end{gathered}
$$

subject to the spin configuration realized at a given time of the simulation and averaged over Monte Carlo steps at times of the equilibrium state simulation; in the last step the calculation of the magnetoresistance is carried out under relation $\delta_{h}=\frac{(J \uparrow-J \downarrow)^{2}}{4 J \uparrow J \downarrow}$.

In Monte Carlo calculations the temperature scale is determined through the value of the exchange integral. So for comparison of the calculated and experimentally measured temperature dependence of the magnetoresistance we used the $a b$ initio determined values of the exchange integral.

More detailed description of Monte-Carlo simulations is presented in our papers [9, 10]. The graphs of calculated temperature dependence of the magnetoresistance are given in Fig. 2, which demonstrate a good agreement with experimental data [1].

\section{Conclusions}

In this paper, magnetic properties of $\mathrm{Ni} / \mathrm{Cu} / \mathrm{Ni} \mathrm{Co} / \mathrm{Cu} / \mathrm{Co}$ and $\mathrm{Fe} / \mathrm{Cr} / \mathrm{Fe}$ trilayer structures was investigated by abinitio methods. The exchange interaction integrals for these structures were first calculated, that allowed us to obtain the temperature dependence of the magnetoresistance, 
which demonstrate a good agreement with experimental data.

Support by RFBR grant N17-02-00279 is acknowledged.

\section{References}

[1] J. Bass and W. P. Pratt, J. Magn. Magn. Mater. 200, 274-289 (1999).

[2] S. N. Piramanayagam and T. C. Chong, Developments in data storage : materials perspective (Wiley-IEEE Press, 2011).

[3] P. G. Kresse and J. Furthmuller, Phys. Rev. B 54, 11169 (1996).

[4] J. P. Perdew, K. Burke and M. Ernzerhof, Phys. Rev. Lett. 77, 3865 (1996).
[5] K. Lejaeghere, V. Van Speybroeck, G. VanOost and S. Cottenier, Critical Reviews in Solid State and Materials Sciences, 39(1), 1-24 (2014).

[6] C. S. Wang and A. J. Freeman, Phys. Rev. B 21, 4585 (1980).

[7] Z. Yang and V. I. Gavrilenko and R Wu, Surf. Sci. 447, 212(2000).

[8] A. Ney and A. Scherz and P. Poulopoulos and K. Lenz and H. Wende and K. Baberschke, Phys. Rev. B 65, 024411 (2001).

[9] V. V. Prudnikov, P. V. Prudnikov, D. E. Romanovskiy, JETP Letters 102, 668-673 (2015).

[10] V. V. Prudnikov, P. V. Prudnikov, D. E. Romanovskiy, J. Phys. D: Appl. Phys. 49(23), 235002 (2016). 